\title{
METODOLOGÍA PARA EL ANÁLISIS DE VULNERABILIDAD ANTE AMENAZAS DE INUNDACIÓN, REMOCIÓN EN MASA Y FLUJOS TORRENCIALES EN CUENCAS HIDROGRÁFICAS
}

\author{
METHODOLOGY FOR ANALYSIS OF VULNERABILITY FOR FLOOD \\ HAZARDS, MASS MOVEMENTS AND WATERSHED TORRENT FLOW
}

Jorge Mario Vera Rodríguez

Adriana Paola Albarracín Calderón**

Fecha de recepción: 27 de septiembre de 2016

Fecha de revisión: 12 de marzo de 2017

Fecha de aprobación: 5 de mayo de 2017

Cómo citar: J. M. Vera Rodríguez y A. P. Albarracín Calderón, "Metodología para el análisis de vulnerabilidad ante amenazas de inundación, remoción en masa y flujos torrenciales en cuencas hidrográficas," Ciencia e Ingeniería Neogranadina, vol. 27, no. 2, p. 109-136. DOI: http://dx.doi.org/10.18359/rcin.2309

\section{RESUMEN}

La gestión del riesgo es un componente fundamental para la ordenación de cuencas hidrográficas y, en general, para la gestión integral del territorio; sin embargo, los ejercicios de gestión del riesgo han sido hegemonizados desde las visiones parciales de las ciencias naturales y aplicadas, por lo que se han centrado principalmente en el análisis de amenazas, y se ha dado menor importancia a los componentes de la vulnerabilidad. Este trabajo esboza una propuesta metodológica para el análisis y la espacialización de la vulnerabilidad ante amenazas de inundación, remoción en masa y flujos torrenciales, soportada en un marco teórico holístico y en el uso de sistemas de información geográfica. Dicha metodología se aplicó a una microcuenca del río Combeima, ubicada en el departamento del Tolima. Los resultados obtenidos permiten verificar su viabilidad práctica, siendo además susceptible de generalización.

\footnotetext{
* Ingeniero Agroindustrial; Msc., doctorando en Planificación y Manejo Ambiental de Cuencas Hidrográficas. Investigador del Grupo Interdisciplinario de Estudios sobre el Territorio, de la Universidad del Tolima, Ibagué, Colombia. Correo electrónico: jmverar@ut.edu.co.ORCID: http://orcid.org/0000-0001-5411-4140

** Ingeniera Forestal, Msc.. Investigadora del Grupo Interdisciplinario de Estudios sobre el Territorio, Universidad del Tolima, Ibagué, Colombia. Correo electrónico: palbarra@ut.edu.co. ORCID: http://orcid.org/0000-0001-6757-121X
} 
Palabras clave: Vulnerabilidad, gestión del riesgo, cuencas hidrográficas, amenaza por inundación, remoción en masa, flujos torrenciales.

\section{ABSTRACT}

Risk management is a fundamental component of the distribution of watersheds and, in general, for the integral administration of the territory. However, risk management exercises have been hegemonized from the partial visions of the natural and applied sciences, so they have focused mainly on the analysis of threats, giving less importance to the components of vulnerability. This work outlines a methodological proposal for the analysis and spatialization of the vulnerability to flooding threat, mass removal and torrential flows, supported by a holistic theoretical framework and the use of geographic information systems. This methodology was applied to a micro basin of the Combeima River, located in the department of Tolima. The obtained results allow verifying its practical viability, being also susceptible of generalization.

Keywords: Vulnerability, risk management, watershed, flood threat, mass removal, torrential flows.

\section{INTRODUCCIÓN}

El riesgo de desastres es una construcción social, relacionada con el potencial de pérdidas o daños que pueden ocurrir a una comunidad, los ecosistemas que le sustentan o sus medios de vida, como resultado de la convolución o mutuo condicionamiento entre amenaza y vulnerabilidad. Estos constituyen lo que se denominan factores de riesgo [1][8], y que en el caso de la vulnerabilidad están fuertemente ligados al modelo de desarrollo.

Un desastre se entiende como la materialización de un riesgo a tal escala que sus impactos superan la capacidad de respuesta o recuperación del sistema ambiental afectado, y por ello requiere apoyo externo. En este sentido, no todo riesgo es un riesgo de desastre, ya que pueden ocurrir eventos en los que los niveles de daños y pérdidas generados no comprometan de manera seria el funcionamiento del sistema; de allí que uno de los objetivos principales de los procesos de gestión de riesgos de desastres consista en la disminución de los niveles de daños o pérdidas probables a niveles aceptables (prevención y mitigación) [9].

La Fig. 1 presenta cómo el riesgo de desastre se da en la medida en que coinciden unas condiciones de amenaza y vulnerabilidad, que son los factores de riesgo, de manera que para una adecuada gestión del riesgo es necesario entender la forma como interactúan dichos factores y configuran situaciones de riesgo concretas que representan posibilidades reales de afectación a comunidades, los ecosistemas que los soportan y sus medios de vida. 


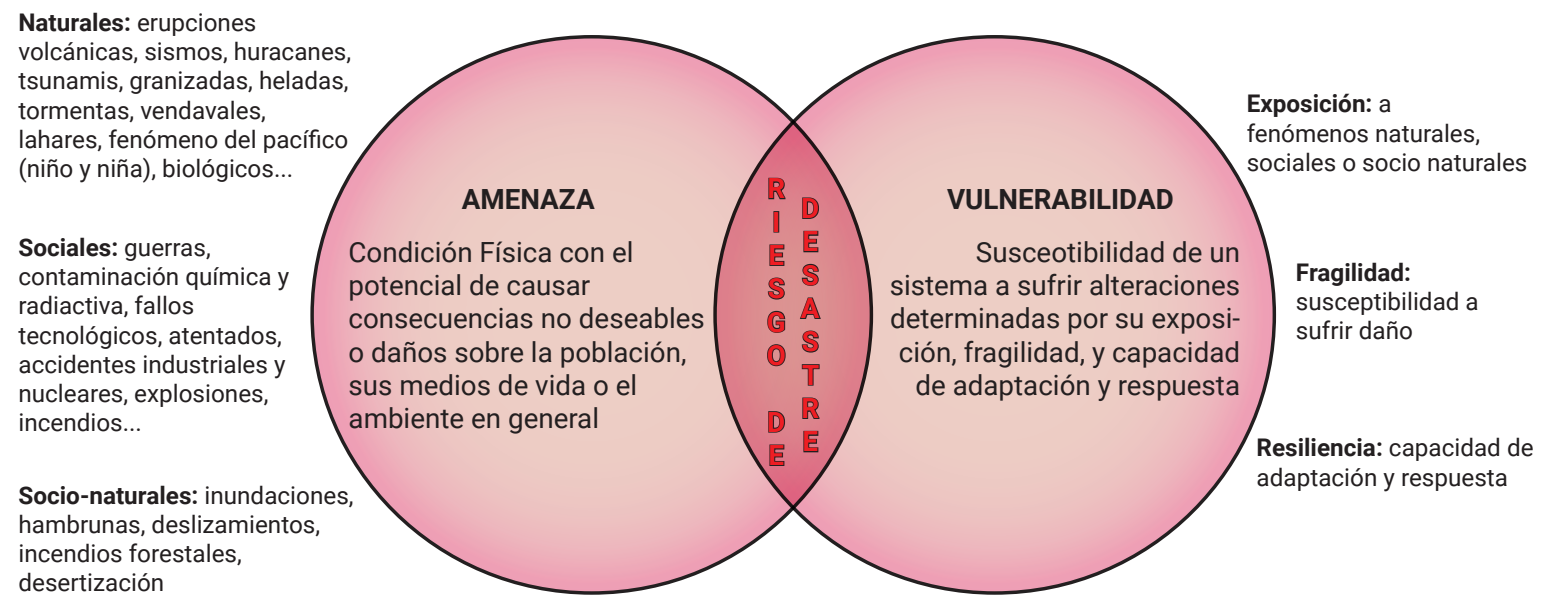

Fig. 1. Factores del riesgo de desastres desde el enfoque holístico

Fuente: elaboración propia.

La amenaza hace referencia a los procesos externos e internos a un sistema, de origen natural, antrópico o socionatural, que interactúan con este y que tienen el potencial de inducir una transformación significativa en él, ya sea de manera lenta o súbita. En este sentido, se refiere a la condición física con el potencial de causar consecuencias no deseables o daños sobre la población, los ecosistemas de que se valen o sus medios de vida. En este punto es necesario resaltar que la mayoría de las configuraciones ambientales o territoriales están expuestas a perturbaciones (amenazas) múltiples, interactuantes [5], [10].

Por su parte, la vulnerabilidad es considerada como un factor interno de riesgo y alude a las características de un sistema desde el punto de vista de su exposición, capacidad para anticipar, sobrevivir, resistir y recu- perarse del impacto de una amenaza natural, antrópica o socionatural, que implica una combinación de factores que determinan el grado al que un sistema se encuentra en riesgo por un evento distinto e identificable de la naturaleza o de la sociedad [11]. La vulnerabilidad tiene un carácter específico según el tipo de amenazas o perturbaciones que influyen en el sistema.

El nivel de vulnerabilidad es determinado por factores asociados al modelo de desarrollo, la disponibilidad y fragilidad de los medios de subsistencia, la sobrepoblación, la cultura, la organización social, la percepción social frente a los riesgos, la capacidad institucional, el equilibrio ambiental, la capacidad de prevención, respuesta o recuperación, entre otros aspectos.

La vulnerabilidad es función de tres componentes principales: la exposición ante 
amenazas naturales, socionaturales o antrópicas; la sensibilidad o fragilidad de los elementos expuestos y la capacidad de adapta- ción o recuperación. La Fig. 2 representa la relación entre los factores de la vulnerabilidad y sus componentes o subsistemas [12].

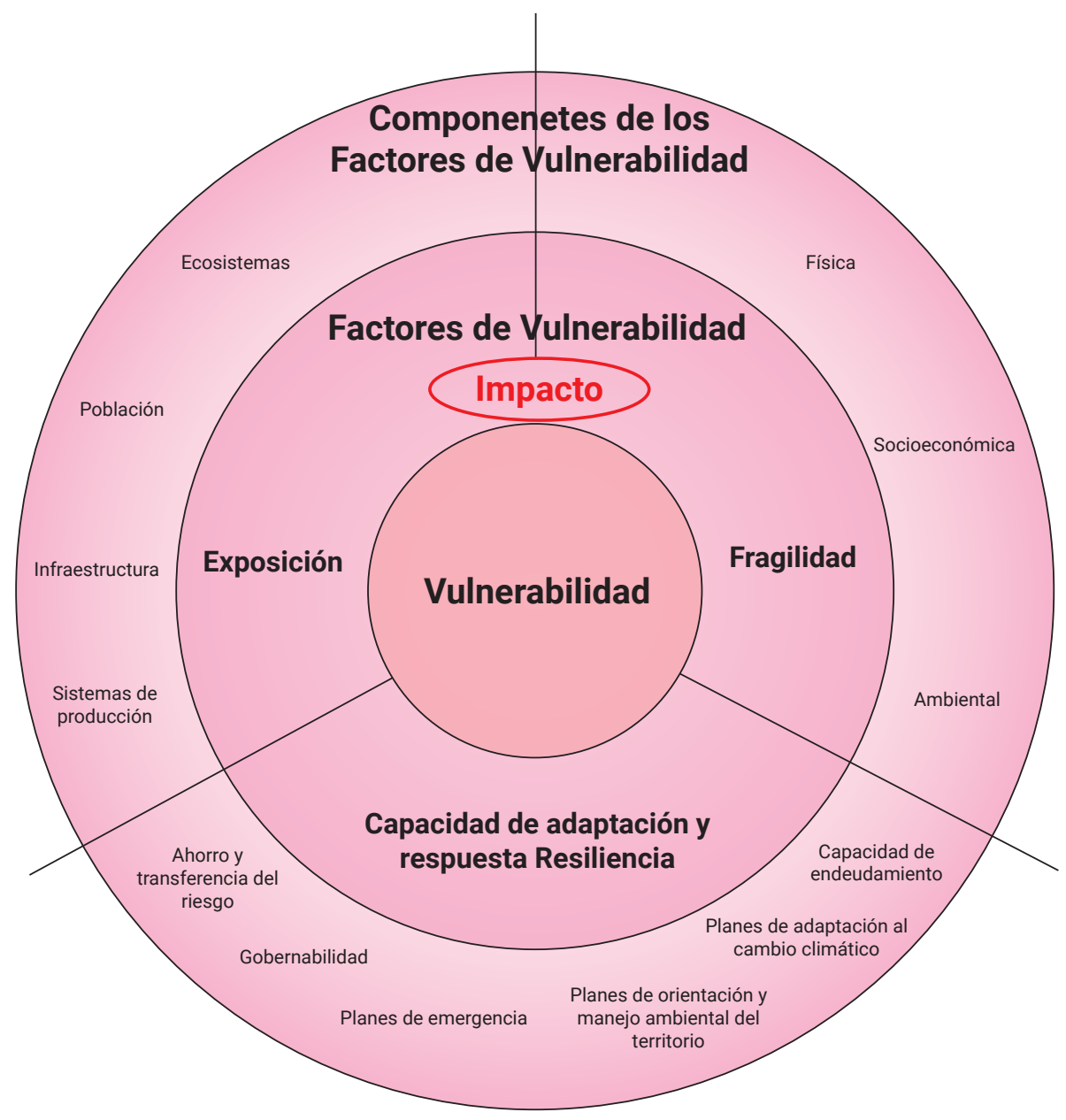

Fig. 2. Factores y componentes de la vulnerabilidad Fuente: elaboración propia a partir de datos de [12].

La Exposición como factor de vulnerabilidad se refiere al nivel donde ecosistemas, población, infraestructuras y sistemas de producción se encuentran en zonas de incidencia potencial de las amenazas consideradas en el análisis.
Por su parte, la fragilidad hace referencia al nivel de susceptibilidad intrínseca de los elementos expuestos a ser afectados por una magnitud estimada de la amenaza; los componentes de la fragilidad son el físico, el socioeconómico y el ambiental. 
La capacidad de respuesta y adaptación tiene que ver con la posibilidad de las comunidades para atender, asumir, recuperarse y adaptarse ante grandes variaciones en sus condiciones normales de vida, producto de un desastre. Esto está determinado por aspectos como niveles de gobernabilidad, nivel cultural, capacidad de ordenación y gestión ambiental del territorio, niveles de preparación y diseño de planes de manejo de emergencias y gestión del riesgo, capacidad de endeudamiento, capacidad de ahorro y transferencia de riesgos mediante pólizas de aseguramiento, entre otros.

Aunque es usual encontrar el uso del término resiliencia para hacer referencia a este componente de la vulnerabilidad, ello es impreciso, ya que esta se refiere fundamentalmente a la tolerancia que tienen las configuraciones territoriales de soportar una acción perturbadora antes de presentar un cambio sustancial en sus características, en tanto que la capacidad de adaptación hace referencia a la posibilidad que tienen los ecosistemas de ajustarse a variaciones sustanciales en su entorno y frente a acciones perturbadoras, súbitas o no, de manera que puedan seguir funcionando a pesar de cambios cualitativos o cuantitativos importantes [13].

Pese a la amplia documentación y legislación sobre gestión del riesgo y planificación de cuencas hidrográficas que plantea incorporar el riesgo como variable clave en la ordenación territorial, la valoración de este generalmente presenta un sesgo, dado que usualmente se hace referencia a las amenazas (más que nada de origen natural), obviando o dando menor prioridad al análisis y espacialización de la vulnerabilidad [14].

Esto es evidente en los planes de ordenación de cuencas, en los que a pesar de los lineamientos del Decreto 1729 de 2002 [15] y las Guías Técnico-Científicas para la Ordenación y Manejo de Cuencas Hidrográficas [16], [17], se trabajó el componente del riesgo sobre todo desde el análisis de amenazas. En este sentido, se carece de modelos para el análisis integral del riesgo y su articulación en los planes de manejo de cuencas, aunque hace falta evaluar los impactos del Decreto 1640 [18], la Ley 1523 [9] y los lineamientos del anexo $B$ de la guía para la formulación de los planes de ordenación y manejo de Cuencas Hidrográficas [19].

Este trabajo presenta una propuesta metodológica que permite la articulación de la gestión integral del riesgo de desastre asociado a eventos de inundación, torrenciales y de remoción en masa, en los procesos de ordenación de cuencas hidrográficas. Dichos eventos, en el caso de Colombia, son los causantes de más del $75 \%$ de las emergencias y más del $80 \%$ del total de víctimas y daños a la infraestructura social y económica, así como a los medios de vida de las comunidades [20].

Como parte del trabajo, se efectúa un ejercicio de aplicación para el caso de la microcuenca de la Quebrada Cay, ubicada en la Cuenca del Río Combeima, municipio de Ibagué, proceso que, a manera de ejemplo, muestra la forma de operativizar la metodología propuesta. 


\section{MATERIALES Y MÉTODOS}

\subsection{Descripción del área de estudio}

La microcuenca de la quebrada Cay, posee una extensión de 1946 hectáreas, que representan el $7 \%$ del área de la cuenca del río Combeima y cuenta con una población de alrededor de 1700 habitantes. Esta microcuenca es una importante fuente hídrica, ya que en esta se encuentra ubicada una bocatoma mediante la cual se captan 300 litros de agua por segundo para el acueducto principal, que abastece el $80 \%$ de la población de Ibagué.

La microcuenca presenta alturas entre 1300 y 2900 m.s.n.m., una pendiente promedio de $29^{\circ}$ y máxima de $79^{\circ}$. La quebrada Cay nace a los 2800 m.s.n.m. y desemboca a los 1300 m.s.n.m.; su cauce principal tiene un recorrido de $8,34 \mathrm{~km}$ y posee un caudal medio anual de $700 \mathrm{l} / \mathrm{s}$. La zona presenta un promedio multianual de precipitaciones del orden de los 1600 a 1700 mm de lluvia. La microcuenca se clasifica como una corriente de agua natural de régimen torrencial acentuado, dado que presenta características morfométricas que la configuran como una subcuenca pequeña que corre por un vaIle estrecho, y presenta caudales en estiaje reducidos; en épocas de lluvia produce crecidas de consideración con grandes acarreos de fondo y en suspensión [21].

Los principales eventos que han impactado a la comunidad están relacionados con deslizamientos localizados que han afectado de forma continua cultivos, caminos veredales, bocatomas e infraestructura en general.

\subsection{Sistema de indicadores para el análisis de la vulnerabilidad}

El sistema de indicadores propuesto se basa en el modelo conceptual planteado, el cual identifica la exposición, fragilidad y capacidad de adaptación y respuesta como factores de vulnerabilidad, los cuales a su vez presentan una serie de componentes. En este sentido, para cada una de las variables se ha establecido una escala de valoración y unas convenciones, como se indica en la Tabla 1.

Tabla 1. Escala de valoración y convenciones para las variables y componentes de los factores de vulnerabilidad.

\begin{tabular}{|l|l|l|}
\hline \multicolumn{1}{|c|}{ Categoría } & \multicolumn{1}{c|}{ Valor } & Convención \\
\hline Bajo & 1 & \\
\hline Medio & 3 & \\
\hline Alto & 5 & \\
\hline
\end{tabular}

Fuente: elaboración propia.

Dado que la vulnerabilidad es función de la exposición, la fragilidad y la capacidad de adaptación y respuesta, se propone estimar la vulnerabilidad global en función de estos tres factores. En este trabajo se ha optado por una relación aritmética simple, que ha sido escogida por su simplicidad y la capacidad de esquematizar el proceso en general y los criterios de aplicación.

La Tabla 2 presenta las funciones propuestas para estimar cada una de las variables consideradas, así como cada uno de los componentes de los factores de vulnerabilidad identificados. 
Ciencia e Ingeniería Neogranadina

Tabla 2. Funciones para la estimación de la vulnerabilidad y sus componentes.

\begin{tabular}{|c|c|}
\hline Tipo de vulnerabilidad & Función \\
\hline Vulnerabilidad global (V) & $\begin{array}{l}\qquad V=\frac{V E+V F+V C A y R}{3} \\
\text { Vulnerabilidad global }(\mathrm{V}) \\
\text { Vulnerabilidad por exposición (VE) } \\
\text { Vulnerabilidad por fragilidad (VF) } \\
\text { Vulnerabilidad determinada por capacidad de adaptación y respuesta } \\
\text { (VCAyR) }\end{array}$ \\
\hline $\begin{array}{l}\text { Vulnerabilidad por exposición } \\
\text { (VE) }\end{array}$ & $\begin{array}{l}\qquad V E=\frac{V E E+V E I+V E S P+V E P}{4} \\
\text { Vulnerabilidad por exposición de los ecosistemas (VEE) } \\
\text { Vulnerabilidad por exposición de la infraestructura (VEI) } \\
\text { Vulnerabilidad por exposición de los sistemas de producción (VESP) } \\
\text { Vulnerabilidad por exposición de la población (VEP). }\end{array}$ \\
\hline $\begin{array}{l}\text { Vulnerabilidad por fragilidad } \\
\text { (VF) }\end{array}$ & $\begin{array}{l}\qquad V F=\frac{V F F+V F S E+V F A+V F I}{4} \\
\qquad V F A=\frac{C P U S+N D E F+I E C C}{3} \\
\text { Vulnerabilidad por fragilidad física (VFF) } \\
\text { Vulnerabilidad por fragilidad socioeconómica (VFSE) } \\
\text { Vulnerabilidad por fragilidad ambiental (VFA) } \\
\text { Vulnerabilidad por fragilidad institucional (VFI) } \\
\text { Conflictos por uso del suelo (CPUS) } \\
\text { Nivel o grado de deforestación (NDEF) } \\
\text { Impacto estimado del cambio climático (IECC). }\end{array}$ \\
\hline $\begin{array}{c}\text { Vulnerabilidad determinada } \\
\text { por la capacidad de adaptación } \\
\text { y respuesta (VCAyR) }\end{array}$ & $\begin{array}{l}\qquad V C A y R=\frac{V C A y R A C E+V C A y R P R+V C A y G G T}{3} \\
\text { Vulnerabilidad determinada por ahorro y capacidad de endeudamiento } \\
\text { (VCAyRACE) } \\
\text { Vulnerabilidad determinada por percepción del riesgo (VCAyRPR) } \\
\text { Vulnerabilidad determinada por la gobernanza y capacidad de gestión } \\
\text { territorial (VCAyGGT) }\end{array}$ \\
\hline
\end{tabular}

Fuente: elaboración propia. 
En las Tablas 3, 4 y 5 se desagregan cada uno de los componentes, variables por medir y criterios de evaluación propuestos para la estimación de la vulnerabilidad por exposición, fragilidad y capacidad de adaptación y respuesta. Dichos criterios y variables han sido establecidos con base en criterios discutidos por los autores con expertos regionales en gestión del riesgo de desastres. En la Tabla 6 se presenta una matriz propuesta para valorar la gobernanza y capacidad de gestión territorial.

Tabla 3. Componentes, variables y criterios de evaluación para determinación de la vulnerabilidad por exposición.

\begin{tabular}{|c|c|c|c|}
\hline Componentes & & Variables de medición & Criterios de evaluación \\
\hline \multirow{5}{*}{$\begin{array}{l}\text { Vulnerabilidad } \\
\text { por exposición de } \\
\text { ecosistemas } \\
\text { (VEE) }\end{array}$} & \multirow{5}{*}{ Localización } & Bosque natural & \multirow{4}{*}{$\begin{array}{l}\text { Más del } 20 \% \text { del total } \\
\text { de los elementos } \\
\text { expuestos en zonas de } \\
\text { nivel de amenaza alta, } \\
\text { vulnerabilidad alta ( } 3 \\
\text { puntos) }\end{array}$} \\
\hline & & Parques naturales & \\
\hline & & Humedales & \\
\hline & & Páramos & \\
\hline & & Reservas naturales & \multirow{4}{*}{$\begin{array}{l}\text { Menos del } 20 \% \text { del total de } \\
\text { los elementos expuestos } \\
\text { en zonas de nivel de } \\
\text { amenaza alta y más del } \\
30 \% \text { en amenaza media, } \\
\text { vulnerabilidad media (2 } \\
\text { puntos) }\end{array}$} \\
\hline \multirow{3}{*}{$\begin{array}{l}\text { Vulnerabilidad } \\
\text { por exposición de } \\
\text { infraestructura } \\
\text { (VEI) }\end{array}$} & \multirow{3}{*}{ Localización } & Vías de comunicación, puentes & \\
\hline & & $\begin{array}{l}\text { Líneas vitales (acueducto, energía } \\
\text { eléctrica, alcantarillado, combustibles) }\end{array}$ & \\
\hline & & $\begin{array}{l}\text { Hospitales, escuelas, ancianatos, } \\
\text { mataderos, etc. }\end{array}$ & \\
\hline $\begin{array}{l}\text { Vulnerabilidad } \\
\text { por exposición de } \\
\text { población } \\
\text { (VEP) }\end{array}$ & Localización & Viviendas & \multirow{2}{*}{$\begin{array}{l}\text { Menos del } 20 \% \text { de } \\
\text { elementos en amenaza } \\
\text { alta y más del } 60 \% \text { del } \\
\text { total de los elementos } \\
\text { expuestos se encuentran } \\
\text { en zonas de nivel } \\
\text { de amenaza baja, } \\
\text { vulnerabilidad baja ( } 1 \\
\text { punto) }\end{array}$} \\
\hline $\begin{array}{l}\text { Vulnerabilidad } \\
\text { por exposición } \\
\text { de sistemas de } \\
\text { producción } \\
\text { (VESP) }\end{array}$ & Localización & $\begin{array}{l}\text { Cultivos, producción pecuaria, } \\
\text { plantaciones forestales, áreas de } \\
\text { producción industrial, minera, comercial } \\
\text { o recreativa. }\end{array}$ & \\
\hline
\end{tabular}

Fuente: elaboración propia. 
Ciencia e Ingeniería Neogranadina

Tabla 4. Componentes, variables y criterios de evaluación para determinación de la vulnerabilidad por fragilidad

\begin{tabular}{|c|c|c|c|}
\hline Componentes & \multicolumn{2}{|c|}{ Variables de medición } & Criterios \\
\hline $\begin{array}{c}\text { Vulnerabilidad por } \\
\text { fragilidad } \\
\text { socioeconómica } \\
\text { (VFSE) }\end{array}$ & $\begin{array}{l}\text { Condiciones de vida } \\
\text { de la población }\end{array}$ & $\begin{array}{l}\text { Promedio de } \\
\text { puntaje de Sisbén III }\end{array}$ & $\begin{array}{l}<50 \text { puntos, vulnerabilidad alta ( } 3 \text { puntos) } \\
50 \text { a } 75 \text { puntos, vulnerabilidad media ( } 2 \\
\text { puntos) } \\
>75 \text { puntos, vulnerabilidad baja ( } 1 \text { punto) }\end{array}$ \\
\hline \multirow{3}{*}{$\begin{array}{l}\text { Vulnerabilidad por } \\
\text { fragilidad ambiental } \\
\text { (VFA) }\end{array}$} & \multirow{3}{*}{$\begin{array}{l}\text { Equilibrio en la } \\
\text { relación sociedad- } \\
\text { naturaleza } \\
\text { (degradación de } \\
\text { base ecosistémica } \\
\text { y prácticas } \\
\text { productivas } \\
\text { inadecuadas, } \\
\text { efectos estimados } \\
\text { del cambio } \\
\text { climático) }\end{array}$} & $\begin{array}{l}\text { Conflictos por uso } \\
\text { del suelo }\end{array}$ & $\begin{array}{l}\text { Área en conflicto }>40 \% \text {, vulnerabilidad } \\
\text { alta ( } 3 \text { puntos) } \\
\text { Área en conflicto } 20,1 \text { a } 40 \%, \\
\text { vulnerabilidad media ( } 2 \text { puntos) } \\
\text { Área en conflicto }<20 \% \text {, vulnerabilidad } \\
\text { baja (1 punto) }\end{array}$ \\
\hline & & $\begin{array}{l}\text { Nivel de } \\
\text { deforestación }\end{array}$ & $\begin{array}{l}\text { Coberturas boscosas < } 20 \% \text { alta, } \\
\text { vulnerabilidad alta ( } 3 \text { puntos) } \\
\text { Coberturas boscosas entre } 20,1 \text { a } 50 \% \text {, } \\
\text { vulnerabilidad media ( } 2 \text { puntos) } \\
\text { Coberturas boscosas }>50 \% \text {, } \\
\text { vulnerabilidad baja ( } 1 \text { punto) }\end{array}$ \\
\hline & & $\begin{array}{l}\text { Variaciones de } \\
\text { temperatura, } \\
\text { precipitación, } \\
\text { pérdida de suelo, } \\
\text { etc. }\end{array}$ & $\begin{array}{l}\text { Impacto estimado alto, vulnerabilidad } \\
\text { alta ( } 3 \text { puntos) } \\
\text { Impacto estimado medio, vulnerabilidad } \\
\text { media ( } 2 \text { puntos) } \\
\text { Impacto estimado bajo, vulnerabilidad } \\
\text { baja (1 punto) }\end{array}$ \\
\hline \multirow[b]{2}{*}{$\begin{array}{c}\text { Vulnerabilidad por } \\
\text { fragilidad física } \\
\text { (VFF) }\end{array}$} & \multirow{2}{*}{$\begin{array}{l}\text { Sismo resistencia, } \\
\text { materiales de } \\
\text { construcción, } \\
\text { características } \\
\text { estructurales } \\
\text { y estado de } \\
\text { mantenimiento }\end{array}$} & Viviendas & \multirow{2}{*}{$\begin{array}{l}\text { Inadecuada (cumple menos de } 2 \\
\text { criterios), vulnerabilidad alta (3 puntos) } \\
\text { Deficiente (cumple } 2 \text { criterios), } \\
\text { Vulnerabilidad media ( } 2 \text { puntos) } \\
\text { Óptima (Cumple } 3 \text { o más criterios), } \\
\text { Vulnerabilidad baja ( } 1 \text { punto) }\end{array}$} \\
\hline & & $\begin{array}{l}\text { Infraestructura } \\
\text { productiva social y } \\
\text { de servicios }\end{array}$ & \\
\hline $\begin{array}{c}\text { Vulnerabilidad por } \\
\text { fragilidad } \\
\text { institucional } \\
\text { (VFI) }\end{array}$ & $\begin{array}{l}\text { Grado de riesgo de } \\
\text { corrupción de las } \\
\text { entidades públicas } \\
\text { locales o regionales }\end{array}$ & $\begin{array}{l}\text { Índice de } \\
\text { transparencia de las } \\
\text { entidades públicas }\end{array}$ & $\begin{array}{l}<60 \text { puntos, vulnerabilidad alta ( } 3 \text { puntos) } \\
60 \text { a } 80 \text { puntos, vulnerabilidad media ( } 2 \\
\text { puntos) } \\
>80 \text { puntos, vulnerabilidad baja ( } 1 \text { punto) }\end{array}$ \\
\hline
\end{tabular}

Fuente: elaboración propia. 
Tabla 5. Componentes, variables y criterios de evaluación para determinación de la vulnerabilidad determinada por capacidad de adaptación y respuesta

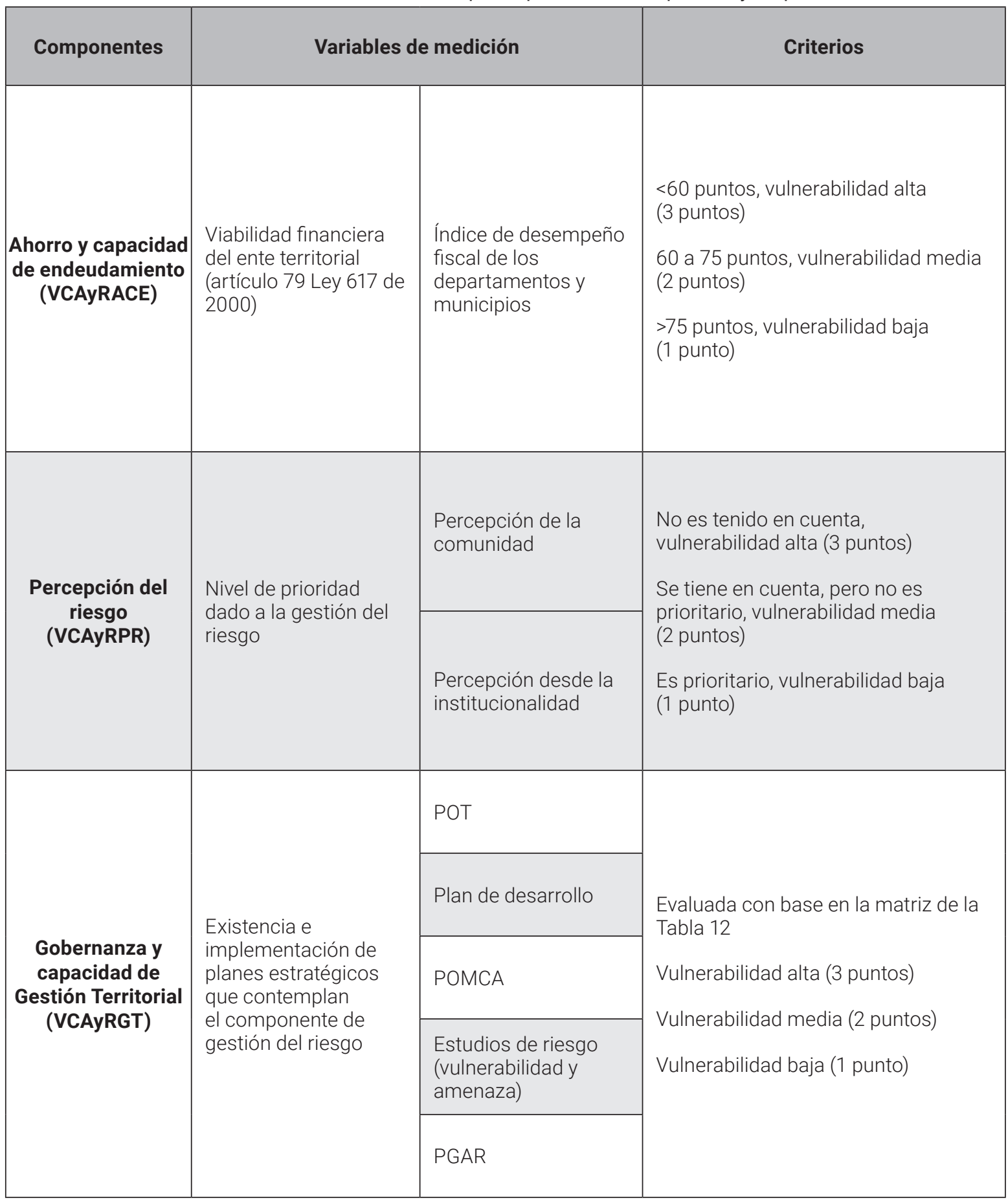

Fuente: elaboración propia. 
Tabla 6. Matriz de evaluación para determinar vulnerabilidad por capacidad de gobernanza y gestión territorial

\begin{tabular}{|c|l|l|l|l|l|}
\hline $\begin{array}{c}\text { Instrumento que } \\
\text { contempla la gestión } \\
\text { del riesgo }\end{array}$ & $\begin{array}{c}\text { No. } \\
\text { cuenta }\end{array}$ & $\begin{array}{c}\text { Desactualizado } \\
\text { y aplica }\end{array}$ & $\begin{array}{c}\text { Desactualizado } \\
\text { y no aplica }\end{array}$ & $\begin{array}{c}\text { Actualizado } \\
\text { y no aplica }\end{array}$ & $\begin{array}{c}\text { Actualizado } \\
\text { y aplica }\end{array}$ \\
\hline $\begin{array}{c}\text { Plan de Ordenamiento } \\
\text { Territorial (POT) }\end{array}$ & Alta & Media & Alta & Baja \\
\hline $\begin{array}{c}\text { Plan de Ordenamiento } \\
\text { Cuencas Ambiental de } \\
\text { (POMCA9 }\end{array}$ & Alta & Media & Alta & Media & Baja \\
\hline $\begin{array}{c}\text { Plan de Gestión Ambiental } \\
\text { Regional (PGAR9 }\end{array}$ & Alta & Media & Alta & Media & Baja \\
\hline Política pública & Alta & Media & Alta & Media & Baja \\
\hline Plan de desarrollo & Alta & Media & Alta & Alta & Media \\
\hline Estudios de riesgo & Alta & Media & Baja \\
\hline
\end{tabular}

Fuente: elaboración propia.

\subsection{Fuentes de información}

En la Tabla 7 se relacionan la información y las fuentes que se tuvieron en cuenta para el cálculo de cada uno de los componentes de la vulnerabilidad.

Tabla 7. Información y fuentes de información que se tomaron en cuenta en el proceso de aplicación de la metodología propuesta.

\begin{tabular}{|l|l|}
\hline \multicolumn{1}{|c|}{ Información } & Fuente \\
\hline $\begin{array}{l}\text { Mapa de amenazas generado durante el proceso de elaboración de Plan de Ordenación y } \\
\text { Manejo de la cuenca mayor del río Coello, escala 1:25000 }\end{array}$ & [22] \\
\hline Mapa de cobertura de la tierra escala 1:10000 & [23] \\
\hline Mapa de amenazas corregimiento 9 de Ibagué & [21] \\
\hline
\end{tabular}




\begin{tabular}{|l|l|}
\hline \multicolumn{1}{|c|}{ Información } & Fuente \\
\hline Mapa de zonas inestables cuenca del río Combeima escala 1:10000 & [23] \\
\hline Mapa de infraestructura corregimiento 9 de Ibagué & [21] \\
\hline Mapa de infraestructura centro poblado Cay & [23] \\
\hline Índice de desempeño fiscal de los departamentos y municipios & [30] \\
\hline $\begin{array}{l}\text { Índice de transparencia de las instituciones públicas (departamentos, contralorías } \\
\text { departamentales y municipios) }\end{array}$ & [29] \\
\hline Inventario de procesos en la cuenca del Combeima 1991 y 2009 elaborado por Ingeominas & [23] \\
\hline Estimación de sedimentos para la cuenca del río Coello, periodo 2011-2030 & [28] \\
\hline Inventario de emergencias y desastres en la cuenca del Combeima & [21]-[23] \\
\hline Modelo de elevación digital Aster versión 2, 30 m de resolución & [31] \\
\hline
\end{tabular}

Fuente: elaboración propia.

\section{RESULTADOSY DISCUSIÓN}

Dado que la vulnerabilidad por exposición (VE) está determinada por el grado de exposición ante las amenazas, es necesario contar con un mapa de amenazas y otro de elementos expuestos, los cuales deben ser contrastados para identificar los niveles de exposición existentes. El proceso partió de la definición del mapa de amenazas de inundación, remoción en masa y flujos torrenciales, que son el tipo de eventos más recurrentes en la microcuenca y generan emergencias en el área de estudio.
A continuación, se presentan las características de estos dos aspectos (amenaza y elementos expuestos), para la microcuenca de la quebrada Cay y posteriormente se efectúa el cruce de estas variables.

\subsection{Amenaza}

Este estudio tuvo como base el mapa de amenazas generado en el marco del estudio de caracterización para el ordenamiento del corregimiento 9 del municipio de Ibagué [21], de lo cual se extrajo el área de la microcuenca de la quebrada Cay, como se observa en la Fig. 3. 
Ciencia e Ingeniería Neogranadina

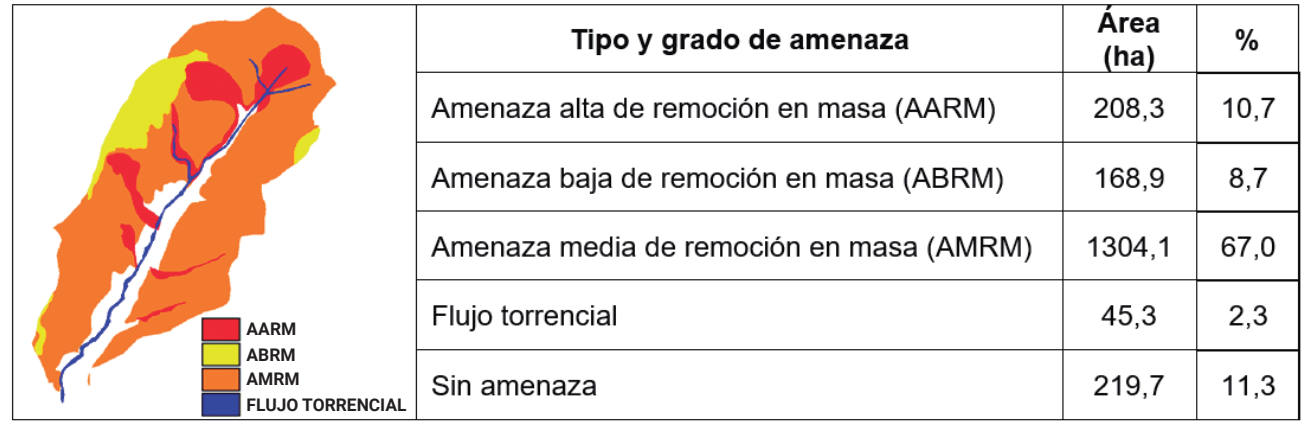

Fig. 3. Tipos y grado de amenaza por inundación, remoción en masa y flujos torrenciales identificados en la microcuenca de la quebrada Cay.

Fuente: elaboración propia a partir de datos de [21].

En primera instancia se consideró trabajar con el mapa de amenazas generado durante el proceso de elaboración del POMCA de la cuenca mayor del río Coello, el cual fue elaborado a escala 1:25000 [22]; sin embargo, al efectuar un análisis detallado de las categorías empleadas para la clasificación de las amenazas, se encontraron deficiencias conceptuales, dado que en el POMCA se define la susceptibilidad como el grado de predisposición de un sitio a que en él se genere un evento $u$ amenaza debido a sus condiciones intrínsecas [22]. En este sentido no existe claridad respecto a qué diferencia existe entre susceptibilidad e inestabilidad y existen criterios que ubican la susceptibilidad más como una vulnerabilidad física que como una amenaza.

\subsection{Vulnerabilidad por exposición}

\subsubsection{Vulnerabilidad por exposición de infraestructura}

La Tabla 8 presenta la matriz relacional empleada para determinar el grado de exposición de los diferentes elementos en la microcuenca, con base en el nivel de amenaza asociado con su localización.

Tabla 8. Matriz de dos dimensiones para determinar la VEI y la VEP en la microcuenca de la quebrada Cay

\begin{tabular}{|l|l|l|l|l|}
\hline \multirow{2}{*}{ Infraestructura } & \multicolumn{3}{|c|}{ Tipo y nivel de amenaza } \\
\cline { 2 - 5 } & $\begin{array}{c}\text { Alta por remoción } \\
\text { en masa (AARM) }\end{array}$ & $\begin{array}{c}\text { Media por remoción } \\
\text { en masa } \\
\text { (AMRM) }\end{array}$ & $\begin{array}{c}\text { Baja por remoción } \\
\text { en masa } \\
\text { (ABRM) }\end{array}$ & $\begin{array}{c}\text { Flujo } \\
\text { torrencial }\end{array}$ \\
\hline Bocatoma acueducto & Alta & Media & Baja & Alta \\
\hline $\begin{array}{l}\text { Centro de } \\
\text { rehabilitación }\end{array}$ & Alta & Media & Baja & Alta \\
\hline
\end{tabular}

Continúa $\rightarrow$ 


\begin{tabular}{|c|c|c|c|c|}
\hline \multirow[b]{2}{*}{ Infraestructura } & \multicolumn{4}{|c|}{ Tipo y nivel de amenaza } \\
\hline & $\begin{array}{l}\text { Alta por remoción } \\
\text { en masa (AARM) }\end{array}$ & $\begin{array}{c}\text { Media por remoción } \\
\text { en masa } \\
\text { (AMRM) }\end{array}$ & $\begin{array}{c}\text { Baja por remoción } \\
\text { en masa } \\
\text { (ABRM) }\end{array}$ & $\begin{array}{l}\text { Flujo } \\
\text { torrencial }\end{array}$ \\
\hline Centro recreacional & Alta & Media & Baja & Alta \\
\hline Comedor comunitario & Alta & Media & Baja & Alta \\
\hline Estación de policía & Alta & Media & Baja & Alta \\
\hline Fábrica & Alta & Media & Baja & Alta \\
\hline Iglesia & Alta & Media & Baja & Alta \\
\hline Institución educativa & Alta & Media & Ваја & Alta \\
\hline Matadero municipal & Alta & Media & Baja & Alta \\
\hline Vía & Alta & Media & Baja & Alta \\
\hline Vivienda & Alta & Media & Baja & Alta \\
\hline
\end{tabular}

Fuente: elaboración propia.

Como puede observarse, se ha determinado que todo aquel elemento que se encuentre en un área con nivel de amenaza alta, media o baja ante eventos de remoción en masa tendrá el mismo nivel de vulnerabilidad por exposición. Por su parte, los elementos que se encuentren en áreas amenazadas por flujos torrenciales tendrán un nivel de vulnerabilidad por exposición alto.

\subsubsection{Vulnerabilidad por exposición de ecosistemas}

En este punto, se trabajó con base en un mapa de coberturas de la tierra que emplea la metodología Corine land cover a escala 1:10.000 [23], mapa en el cual se estimó que dentro de los tipos de ecosistemas considerados, la microcuenca de la quebrada Cay posee una extensión de 914,6 ha de bosque natural fragmentado, correspondientes al $47 \%$ de la extensión de la microcuenca; correspondiendo el $53 \%$ a la sumatoria de áreas construidas, cultivos (permanentes, semipermanentes 0 transitorios) o pastos (manejados o enmalezados). Además, se identificó que no hay presencia de otros tipos de ecosistemas especiales (páramos, humedales, parques naturales y reservas naturales). Con base en la Tabla 3 se determina que la VEE es media.

\subsubsection{Vulnerabilidad por exposición de sistemas de producción}

Para la implementación de esta variable se trabaja con base en cuatro tipologías de co- 
berturas de la tierra tenidas en cuenta en la metodología de clasificación Corine land cover. Estas son: cultivos permanentes, cultivos anuales o transitorios, pastos limpios que se asocian a la ganadería intensiva y pastos enmalezados o enrastrojados, que de acuerdo con la comunidad, en la microcuenca se asocian con la ganadería extensiva. En este sentido, se identificó que el 91,2\% de los sistemas de producción presentan algún grado de exposición ante eventos de remoción en masa o torrenciales, siendo predominante el nivel de exposición medio; por esta razón, al aplicar los criterios propuestos en la Tabla 8 se estima que la VESP es media. La Fig. 4 presenta los resultados de la VEI, VEE y VESP.

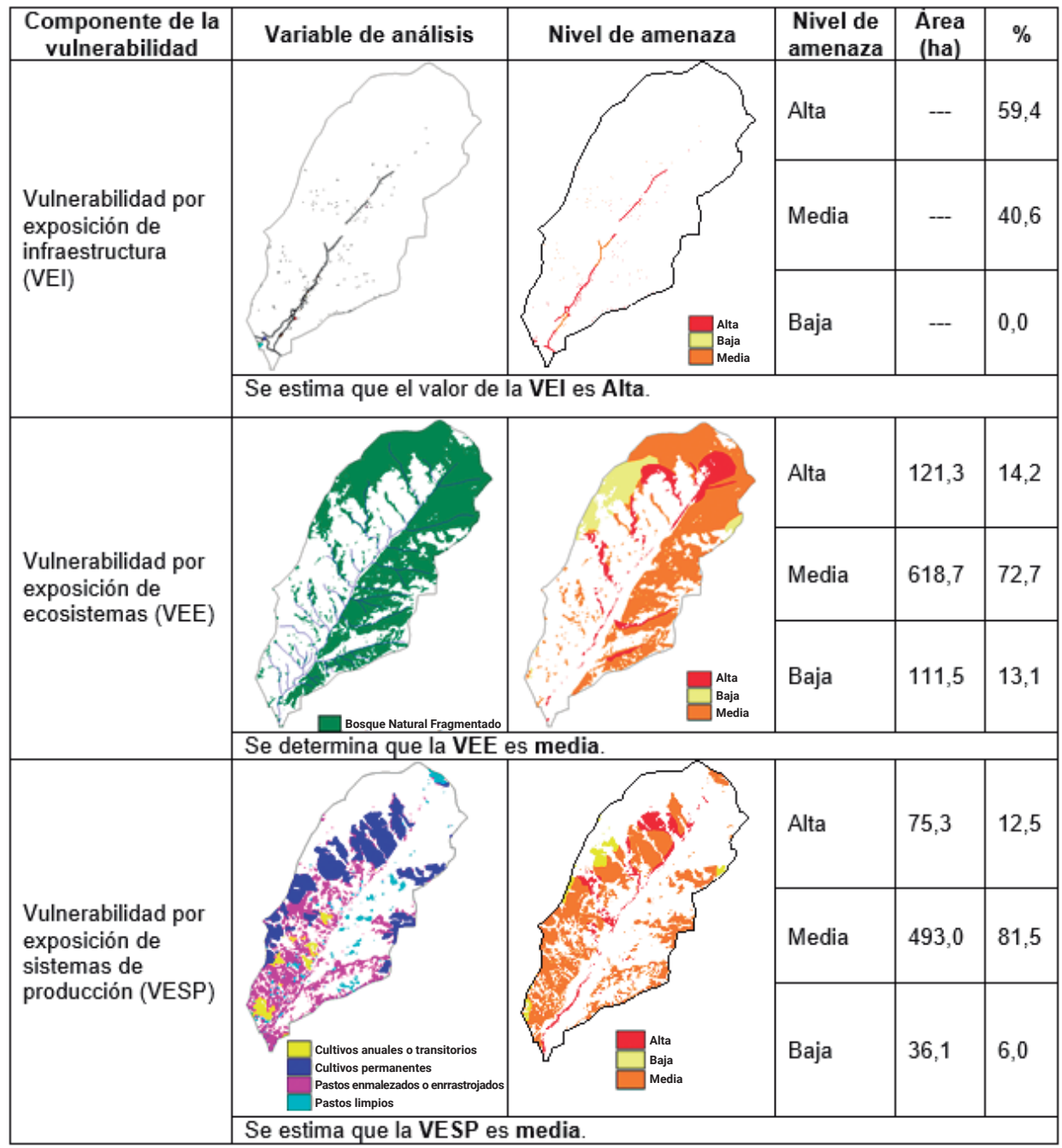

Fig. 4. Resultados de la espacialización de VEI, VEE y VESP

Fuente: elaboración propia. 


\subsubsection{Vulnerabilidad por exposición de población}

Para el manejo de esta variable, se tomó como base la localización de las viviendas en el área de estudio. Al aplicar los criterios propuestos en la Tabla 3, y teniendo en cuenta que más del $30 \%$ de las viviendas se encuentra en áreas con nivel de amenaza alta, se determina que la VEP es alta. En este caso, debido al tamaño de las viviendas, la cartografía no es muy apropiada para representar los resultados a esta escala, por lo que estos se presentan en la Tabla 9.

Tabla 9. Nivel de amenaza a las que se encuentran expuestas las viviendas existentes en la microcuenca de la quebrada Cay

\begin{tabular}{|c|c|}
\hline Nivel de exposición & $\%$ \\
\hline Alta & 35,2 \\
\hline Baja & 3,8 \\
\hline Media & 61,0 \\
\hline
\end{tabular}

Fuente: elaboración propia.

\subsection{Vulnerabilidad por fragilidad}

\subsubsection{Cálculo de la vulnerabilidad por fragilidad socioeconómica}

El cálculo de este indicador se efectúa para toda la población de la vereda a través del valor promedio del puntaje de Sisbén III. Se ha optado por este indicador dado que es empleado para la focalización de la pobla- ción beneficiaria de los programas sociales del Estado. Según [24] dicho indicador está basado en el enfoque de pobreza multidimensional y se calcula con base en una encuesta de 96 preguntas, por medio de la cual se identifica la población con necesidades básicas insatisfechas; por lo tanto, el Estado tiene la obligación de subsidiarles derechos fundamentales, como salud, vivienda, educación, alimentación, entre otros. Los resultados obtenidos se presentan en la Tabla 10 y permiten establecer que el nivel de la VFSE para la población de la microcuenca de la quebrada Cay es alta.

Tabla 10. Valores promedio de los puntajes de Sisbén III para los habitantes de las veredas de la microcuenca de la quebrada Cay

\begin{tabular}{|l|l|l|}
\hline \multicolumn{1}{|c|}{ Nombre } & \multicolumn{1}{|c|}{ Puntaje } & \multicolumn{1}{c|}{ VFSE } \\
\hline La Cascada & 20,12 & Alta \\
\hline Cay P/A & 23,64 & Alta \\
\hline Chapetón & 20,35 & Alta \\
\hline La Victoria & 20,22 & Alta \\
\hline Cay & 31,62 & Alta \\
\hline Ibagué & 24,72 & Alta \\
\hline El Gallo & 20,05 & Alta \\
\hline La Coqueta & 21,38 & Alta \\
\hline ZU Chapetón & 40,31 & Alta \\
\hline
\end{tabular}

Fuente: elaboración propia. 


\subsubsection{Cálculo de la vulnerabilidad por fragilidad ambiental}

La vulnerabilidad por fragilidad ambiental está dada por el promedio aritmético del puntaje de conflictos por uso del suelo, los niveles de deforestación y los impactos estimados del cambio climático.

\section{- Conflictos por uso del suelo}

Para la generación del mapa de conflictos por uso del suelo se tuvieron en cuenta los siguientes criterios:

1. El artículo tercero del Decreto 1449 de 1977 establece respecto a las áreas forestales protectoras: áreas de $100 \mathrm{~m}$ alrededor de los nacimientos de los cuerpos de agua, margen de $30 \mathrm{~m}$ paralelos a los cauces de los cuerpos de agua; terrenos con pendientes superiores al $100 \%\left(45^{\circ}\right)$ [25].

2. El artículo 83 inciso d. del Código Nacional de los Recursos Naturales Renovables [26] designa a las áreas mencionadas en el inciso anterior como propiedad inalienable, imprescriptible e inembargable del Estado.

3. Las áreas con un nivel de erosión actual fuerte son consideradas áreas de recuperación y manejo ambiental, y aquellas donde la erosión es severa son consideradas áreas forestales protectoras.

4. Las áreas con alturas mayores a 2500 m.s.n.m. se recomiendan como áreas forestales protectoras.

5. Las áreas con niveles de inestabilidad media se deben destinar para recupera- ción y manejo, y las de inestabilidad alta se recomiendan como áreas forestales protectoras.

6. Finalmente, las áreas que no cumplan ninguna de las anteriores restricciones se consideran aptas para producción sustentable.

7. En caso de que las áreas de bosque existentes no necesariamente deban ser destinadas a zonas forestales protectoras, 0 como de recuperación y manejo, se debe orientar su uso sustentable como zona forestal protectora-productora, de manera que no se presente pérdida de coberturas boscosas.

8. Para determinar las áreas en conflicto por uso, se contrastan los mapas de uso y cobertura actual de la tierra y el mapa obtenido de usos recomendados, de manera que las áreas que tengan un uso actual diferente del recomendado se consideran áreas en conflicto por uso.

Para determinar los niveles de conflicto existentes al contrastar los mapas de coberturas de la tierra actuales y usos del suelo recomendados, es necesario contar con unos criterios que permitan establecer en cada cruce posible cuál es el grado del conflicto. En este sentido, la Tabla 11 presenta la matriz de dos dimensiones construidas para ordenar los criterios de decisión, en tanto que la Fig. 5 muestra los resultados obtenidos. 
Tabla 11. Matriz de dos dimensiones para determinar conflictos por uso en la microcuenca de la quebrada Cay

\begin{tabular}{|c|c|c|c|}
\hline \multirow{2}{*}{ Cobertura } & \multicolumn{3}{|c|}{ Usos recomendados } \\
\hline & $\begin{array}{l}\text { Forestal } \\
\text { protectora }\end{array}$ & $\begin{array}{l}\text { Producción } \\
\text { sustentable }\end{array}$ & $\begin{array}{c}\text { Recuperación } \\
\text { y manejo }\end{array}$ \\
\hline Bosque natural fragmentado & Sin conflicto & Sin conflicto & Sin conflicto \\
\hline Cultivos anuales o transitorios & Alto & Sin conflicto & Alto \\
\hline Cultivos permanentes & Alto & Sin conflicto & Medio \\
\hline Pastos enmalezados o enrastrojados & Medio & Bajo & Sin conflicto \\
\hline Pastos limpios & Alto & Bajo & Medio \\
\hline Red vial & Medio & Sin conflicto & Sin conflicto \\
\hline Ríos & Sin conflicto & Sin conflicto & Sin conflicto \\
\hline Tejido discontinuo & Alto & Sin conflicto & Alto \\
\hline Tierras desnudas o degradadas & Alto & Alto & Alto \\
\hline Vegetación secundaria o en transición & Medio & Sin conflicto & Sin conflicto \\
\hline
\end{tabular}

Fuente: elaboración propia. 
Ciencia e Ingeniería Neogranadina

\begin{tabular}{|c|c|c|c|}
\hline \multirow[t]{11}{*}{ Uso actual } & & Área (ha) & $\%$ \\
\hline & Bosque natural fragmentado & 914,3 & 47,0 \\
\hline & Pastos enmalezados o enrastrojados & 324,3 & 16,7 \\
\hline & Cultivos anuales o transitorios & 302,5 & 15,5 \\
\hline & Vegetación secundaria o en transición & 295,8 & 15,2 \\
\hline & Cultivos permanentes & 43,4 & 2,2 \\
\hline & Pastos limpios & 23,1 & 1,2 \\
\hline & Red vial & 16,8 & 0,9 \\
\hline & Ríos & 15,8 & 0,8 \\
\hline & Tejido discontinuo & 7,3 & 0,4 \\
\hline & Tierras desnudas o degradadas & 2,8 & 0,1 \\
\hline \multirow[t]{3}{*}{ Uso recomendado } & Producción sustentable & 10515 & 540 \\
\hline & Forestal protectora & 654,0 & 33,6 \\
\hline & Recuperación y manejo & 241,1 & 12,4 \\
\hline \multirow[t]{5}{*}{ Conflictos por uso } & Alto & 1252 & 60 \\
\hline & & & \\
\hline & Medio & 126,7 & 6,5 \\
\hline & Bajo & 159,3 & 8,2 \\
\hline & Sin conflicto & 1534,8 & 78,9 \\
\hline
\end{tabular}

Fig. 5. Usos del suelo actual, recomendado y conflictos por uso del suelo en la microcuenca de la quebrada Cay

Fuente: elaboración propia.

Con base en los criterios propuestos y dado que el área en conflictos por uso representa el $21,1 \%$ del área de la microcuenca y que de esta el $61,3 \%$ se encuentra en niveles de conflicto medio o alto, se determina que este componente de la vulnerabilidad ambiental es medio.

\section{- Nivel de deforestación}

El decreto 1791 de 1996 establece en su artículo 4 [27] que los propietarios de predios de más de 50 hectáreas deberán mantener en cobertura forestal por lo menos un $10 \%$ de su extensión. De igual forma, 
en el artículo 5 se establece que en terrenos baldíos adjudicados mayores de 50 hectáreas el propietario tendrá mantener una proporción del $20 \%$ de la extensión del terreno en cobertura forestal. De aquí se puede interpretar que desde la perspectiva de la legislación existe un mínimo de coberturas boscosas para garantizar la sustentabilidad de los territorios, el cual oscila entre el $10 \%$ y el $20 \%$ del área de la cuenca. En este sentido, si el territorio cuenta con menos de $20 \%$ de cobertura boscosa la vulnerabilidad por los niveles de deforestación es alta. Con base en criterio experto, se determina que si la deforestación está entre el $20 \%$ y $50 \%$, la vulnerabilidad es media y si es menor al $20 \%$ es baja. Como se observa en la Fig. 5, el nivel de cobertura boscosa representada en bosque natural fragmentado es del $47 \%$, por lo que la vulnerabilidad es media.

\section{- Impacto estimado del cambio climático}

En 2013 se efectuó una modelación para la determinar la producción de sedimentos desde contextos de cambio climático en la cuenca hidrográfica del río Coello, de la cual hace parte la microcuenca de la quebrada Cay [28]. Para este propósito se tomó el periodo de referencia 1988-2007, y para estimar la influencia del cambio climático, el periodo 2011-2030. El modelo empleado estimó la pérdida de suelo en la cuenca desde escenarios de cambio climático, con base en los tipos de cobertura y uso actual del suelo, tipos de suelo y relieve, identificando aquellas unidades territoriales más susceptibles a los procesos de erosión. La Tabla 12 presenta parte de los resultados obtenidos, los cuales permiten determinar que el nivel del impacto estimado en cuanto al cambio climático en la microcuenca de la quebrada Cay es alto.

Tabla 12. Estimación de sedimentos (ton/ha/año) según subcuencas en la cuenca mayor del río Coello para los escenarios 1988-2007 y 2011-2030.

\begin{tabular}{|c|c|c|c|c|}
\hline Subcuencas & 1988-2007 & $\begin{array}{l}\text { Clasificación } \\
\text { grado pérdida } \\
\text { suelos /erosión }\end{array}$ & 2011-2030 & $\begin{array}{c}\text { Clasificación grado } \\
\text { pérdida } \\
\text { suelos /erosión }\end{array}$ \\
\hline Combeima parte alta & 17,44 & Moderada & 8,5 & Moderada \\
\hline Toche parte media & 11,51 & Moderada & 13,27 & Moderada \\
\hline Combeima parte media & 274,16 & Muy Alta & 147,06 & Alta \\
\hline Cay & 65,09 & Alta & 60,49 & Alta \\
\hline Coello parte media & 43,76 & Moderada & 37,03 & Moderada \\
\hline Coello parte media & 267,25 & Muy alta & 267,19 & Muy alta \\
\hline
\end{tabular}

Fuente: elaboración propia a partir de datos de [28]. 


\subsubsection{Cálculo de la vulnerabilidad por fragilidad física}

La evaluación de este componente es de tipo cualitativo y el nivel del índice se asigna con base en los siguientes criterios:

1. Las diversas edificaciones existentes en la microcuenca fueron construidas antes de 1998, por lo que no cumplen los parámetros de sismorresistencia de las normas técnicas respectivas NTC-98 y NTC-2010.

2. La mayor parte de las estructuras son de uno o dos niveles (pisos), razón por la cual se estima que su nivel de cimentación es superficial.

3. Se identifica que aproximadamente el $49 \%$ de las viviendas están construidas en bloque o ladrillo, el $27 \%$ están hechas en bahareque y el $24 \%$, en tabla.

4. La población para acceder a la microcuenca solo cuenta con una vía que se encuentra en regular estado en la mayor parte de los tramos.

5. En general, el estado de mantenimiento de las infraestructuras presentes en la microcuenca (viviendas, vías, instituciones educativas, acueducto y redes eléctricas) es inadecuado.

Con base en estos aspectos, se determina que la condición de las infraestructuras es inadecuada, por lo que la VFF el alta.

\subsubsection{Cálculo de la vulnerabilidad por fragilidad institucional}

Se toma como base el índice de transparencia de las entidades públicas [29]. Para el periodo 2015-2016, este arrojó como resultado un valor de 62,2 para Ibagué; 73,5 para el departamento del Tolima y 62,8 para la contraloría departamental. Estas puntuaciones ubican las tres instituciones en un nivel de riesgo de corrupción medio.

Por otro lado, un sondeo a nivel de las Juntas de Acción Veredal permite corroborar que entre 2010 y 2015 no ha habido una variación significativa respecto de los niveles de partición de la comunidad en este escenario organizativo. Con base en lo anterior, se establece que la VFI es medio.

Al computar los puntajes respectivos de cada uno de los componentes de la vulnerabilidad por fragilidad VF, se encuentra que el resultado es de 4,2 puntos, lo que la ubica como vulnerabilidad alta.

\subsection{Vulnerabilidad por capacidad de adaptación y respuesta}

La VCAyR está determinada por la capacidad de ahorro y endeudamiento de los entes territoriales, la percepción social del riesgo institucional y comunitario, y por la capacidad de gobernanza y gestión territorial. A continuación, se presentan los criterios empleados y los resultados obtenidos para la evaluación de la VCAyR.

\subsubsection{Ahorro y capacidad de endeudamiento}

El Departamento Nacional de Planeación [30] efectúa cada año una evaluación del despeño fiscal de los entes territoriales (departamentos y municipios). Este indicador 
evalúa seis variables: 1) autofinanciamiento de gastos de funcionamiento; 2) respaldo del servicio de la deuda; 3 ) dependencia de transferencias y regalías; 4) generación de recursos propios; 5) magnitud de la in- versión, y 6) capacidad de ahorro. La Tabla 13 presenta los resultados obtenidos para el departamento del Tolima y para Ibagué, con base en los cuales se determina que la VCAyRACE es media.

Tabla 13. Indicador de desempeño fiscal Gobernación del Tolima y Alcaldía de Ibagué para 2013

\begin{tabular}{|l|c|c|c|c|c|c|c|l|}
\hline $\begin{array}{c}\text { Ente } \\
\text { territorial }\end{array}$ & 1 & 2 & 3 & 4 & 5 & 6 & $\begin{array}{c}\text { Indicador } \\
\text { fiscal }\end{array}$ & $\begin{array}{c}\text { Ranking } \\
\text { nacional }\end{array}$ \\
\hline Tolima & 82,35 & 2,17 & 73,90 & 84,45 & 82,03 & 27,40 & 62,22 & 26 \\
\hline Ibagué & 45,20 & 10,64 & 66,99 & 87,63 & 86,72 & 53,97 & 76,03 & 20 \\
\hline
\end{tabular}

Fuente: elaboración propia a partir de datos de [30].

\subsubsection{Percepción del riesgo}

Al realizar un ejercicio con 42 habitantes, dentro de los que se encontraban varios líderes sociales de la microcuenca, se les solicitó que organizaran en orden de importancia los temas prioritarios que debían ser atendidos por la institucionalidad. Así, se encontró que los aspectos relacionados explícitamente con el riesgo de desastres están en el nivel medio, como lo evidencia la Tabla 14.

Tabla 14. Orden de importancia que la comunidad considera como los principales problemas que deben ser atendidos por los organismos estatales en la microcuenca de la quebrada Cay

\begin{tabular}{|l|l|}
\hline \multicolumn{1}{|c|}{ Problema } & \multicolumn{1}{c|}{ Prioridad } \\
\hline Mercadeo de los productos agropecuarios & Alta \\
\hline Altos costos de producción & Alta \\
\hline Mal estado de vías de penetración y de caminos vecinales & Alta \\
\hline Mal estado de las viviendas & Media \\
\hline Inestabilidad de los terrenos, constantes deslizamientos y avalanchas & Media \\
\hline Deficiencia en la atención en salud & Media \\
\hline Manejo de basuras & Media \\
\hline Deforestación & Baja \\
\hline
\end{tabular}

Fuente: elaboración propia. 
2.4.3. Capacidad de gobernanza

y gestión del territorio

Para evaluar este componente de la capacidad de adaptación y respuesta se aplicó la matriz propuesta en la Tabla 6 y se obtuvieron los resultados presentados en la Tabla 15. Al promediar los puntajes asignados, se encontró que este componente de la vulnerabilidad es medio.

Tabla 15. Matriz de evaluación para determinar vulnerabilidad por capacidad de gobernanza y gestión territorial para el caso de la microcuenca de la quebrada Cay

\begin{tabular}{|l|l|l|l|l|l|}
\hline $\begin{array}{c}\text { Instrumento que } \\
\text { contempla la gestión } \\
\text { del riesgo }\end{array}$ & $\begin{array}{c}\text { No } \\
\text { cuenta }\end{array}$ & $\begin{array}{c}\text { Desactualizado } \\
\text { y aplica }\end{array}$ & $\begin{array}{c}\text { Desactualizado } \\
\text { y no aplica }\end{array}$ & $\begin{array}{c}\text { Actualizado } \\
\text { y no aplica }\end{array}$ & $\begin{array}{c}\text { Actualizado } \\
\text { y aplica }\end{array}$ \\
\hline POT & Media (3) & Media (3) & & Media (3) & \\
\hline POMCA & & & Alta (5) & & \\
\hline PGAR & & & & Media (3) & \\
\hline Política pública & & & & & \\
\hline Estudios de riesgo & & & & & \\
\hline
\end{tabular}

Fuente: elaboración propia.

Como se evidencia en la Fig. 6, los resultados obtenidos indican que el nivel de vulnerabilidad ante eventos de inundación, remoción en masa y flujos torrenciales en la microcuenca de la quebrada Cay es medio. Adicionalmente, como elemento de visualización del escenario, la Fig. 6 permite identificar cuáles son aquellos componentes 0 subcomponentes de la vulnerabilidad que deben ser intervenidos con el propósito de aumentar la capacidad de respuesta y adaptación de la microcuenca como sistema. Este instrumento permite la focalización de los componentes más relevantes, y se facilita así el diseño de los procesos de gestión. 


\begin{tabular}{|c|c|c|c|c|c|c|c|}
\hline $\begin{array}{c}\text { Componentes de la } \\
\text { vulnerabilidad }\end{array}$ & \multicolumn{2}{|c|}{ Variable } & \multicolumn{3}{|c|}{ Puntaje } & Nivel & $\begin{array}{l}\text { Total } \\
\text { (v) }\end{array}$ \\
\hline \multirow{4}{*}{ VE } & \multicolumn{2}{|c|}{ VEI } & \multicolumn{2}{|c|}{5} & \multirow{4}{*}{4.0} & \multirow{4}{*}{ Media } & \multirow{4}{*}{ M } \\
\hline & & & & 3 & & & \\
\hline & \multicolumn{2}{|c|}{ VEP } & \multicolumn{2}{|c|}{5} & & & \\
\hline & \multicolumn{2}{|c|}{ VESP } & \multicolumn{2}{|c|}{3} & & & \\
\hline \multirow{6}{*}{ VF } & \multicolumn{2}{|c|}{ VFSE } & \multicolumn{2}{|c|}{5} & \multirow{6}{*}{4.2} & \multirow{6}{*}{ Alta } & \\
\hline & \multirow{3}{*}{ VFA } & CPUS & 3 & \multirow{3}{*}{3.7} & & & \multirow{3}{*}{ D } \\
\hline & & NDEF & 3 & & & & \\
\hline & & ICC & 5 & & & & \\
\hline & \multicolumn{2}{|l|}{ VFF } & & 5 & & & \multirow{5}{*}{ A } \\
\hline & VFI & & & 3 & & & \\
\hline \multirow{3}{*}{ VCAyR } & \multicolumn{2}{|c|}{ VCAyRACE } & & 3 & \multirow{3}{*}{3} & \multirow{3}{*}{ Media } & \\
\hline & \multicolumn{2}{|c|}{ VCAyRPR } & & 3 & & & \\
\hline & \multicolumn{2}{|c|}{ VCAyRGT } & & $B$ & & & \\
\hline
\end{tabular}

Fig. 6. Consolidado de los resultados obtenidos en la estimación de la vulnerabilidad (V) y sus componentes en la microcuenca de la quebrada Cay

Fuente: elaboración propia.

Los resultados obtenidos tienen varias fuentes de incertidumbre que es necesario resaltar. La primera de ellas tiene que ver con los márgenes de error en la generación de la información secundaria indicada en la Tabla 7, o con las fuentes específicas empleadas para cada caso en particular. Otra fuente de incertidumbre tiene que ver con la forma de definición de la ponderación y cómputo de las variables y componentes de la vulnerabilidad, es decir, con la estructura del modelo, que en este caso en particular se efectuó con base en criterio experto; sin embrago, dado que el propósito de este trabajo es el planteamiento metodológico general y ejemplificar una forma de aplicación, el cálculo de la incertidumbre asociada escapa a los alcances del mismo.

\section{CONCLUSIONES}

A partir de un modelo conceptual holístico de los factores de riesgo de desastre (amenaza y vulnerabilidad), es posible enriquecer los procesos de ordenación de cuencas hidrográficas. De igual forma, se puede adoptar una serie de indicadores de vulnerabilidad ante amenazas de inundación, remoción en masa y flujos torrenciales, basados en fuentes de información y metodologías de análisis aplicables a cualquier cuenca hidrográfica del país, de manera que se puedan efectuar comparaciones y generalizaciones.

Sin embargo, como punto de partida es necesaria una solidez conceptual que sustente los procesos de identificación, caracterización y espacialización del riesgo de desastres, de manera que estos ejercicios 
tengan una coherencia y profundidad que los constituya en soporte para la toma de decisiones.

El proceso desarrollado en este trabajo permite verificar que es posible adoptar metodologías para la espacialización de la vulnerabilidad, susceptibles de generalización, que permitan superar los sesgos basados en la identificación de las amenazas y llegar a una visión del desastre como construcción social y, por lo tanto, susceptible de gestión integral.

La metodología propuesta presenta ventajas importantes como la implementación de variables mediante indicadores generalizables, el uso del puntaje del Sisbén III como fuente de información estandarizada, pública y actualizada permanentemente, que facilita ejercicios de comparación, al tiempo que permite ahorrar recursos y tiempo, así como el uso de sistemas de información geográfica (SIG) para facilitar la toma de decisiones alrededor de la gestión integral del riesgo de desastres a nivel de cuencas hidrográficas y en general en cualquier ámbito territorial.

De igual forma, esta propuesta posibilita trascender la visión parcial de las ciencias naturales o de las ciencias aplicadas que han sido hegemónicas en la gestión del riesgo, para llegar a una visión holística que articula miradas y herramientas de las ciencias naturales, aplicadas y sociales hacia un abordaje inter y transdisciplinar, en el que todas ellas son enriquecidas por las perspectivas de las otras disciplinas.

La metodología facilita además la articulación de la vulnerabilidad y no solo de las amenazas a los procesos de ordenación de cuencas hidrográficas para la gestión integral del riesgo; de igual forma, facilita desagregar los componentes de la vulnerabilidad para identificar aquellos de mayor incidencia en los que debe centrarse la gestión. En este sentido, y como ejemplo, en el ejercicio de aplicación se observa cómo aunque dos componentes críticos son la exposición de infraestructura y de población, ello no implica necesariamente la reubicación de todos los elementos expuestos, sino que se puede trabajar en disminuir la fragilidad física por parte de dichos elementos; es decir, la prevención a través de la mejora en el diseño y construcción a través de la aplicación de normas como las de sismorresistencia, las cuales reducen los impactos de las vulnerabilidades por exposición de infraestructura y población.

Finalmente, ya que el propósito de este trabajo era aportar una propuesta holística, generalizable y operativa, es necesario evaluarla mediante su aplicación a diversos casos, de manera que se pueda identificar la relación matemática más adecuada entre variables y componentes de la vulnerabilidad, así como el grado de incertidumbre asociado al modelo adoptado.

\section{AGRADECIMIENTOS}

Los autores expresan su agradecimiento a los profesores Alberto Núñez Tello, Miguel Thomas y Héctor Cárdenas, expertos en gestión del riesgo de desastres, quienes aportaron de manera significativa en la definición de los criterios y variables por valorar para la estimación de la vulnerabilidad. De igual forma, fue fundamental el apoyo de la Maestría en Planificación y Manejo Ambiental de 
Cuencas Hidrográficas, de la Universidad del Tolima, al facilitar condiciones logísticas y técnicas para adelantar esta investigación.

\section{REFERENCIAS}

[1] G. Wilches. Auge, caída y levantada de Felipe Pinillo, mecánico y soldador o Yo voy a correr el riesgo. Lima, Perú: Red de Estudios Sociales en Prevención de Desastres en América Latina, 1998.

[2] L. Narváez, A. Lavell y G. Pérez. La gestión del riesgo. Un enfoque basado en procesos. Lima, Perú: Comunidad Andina. Secretaría General; Proyecto Apoyo a la Prevención de Desastres en la Comunidad Andina (Predecan), 2009.

[3] A. Maskrey (Comp.). Los desastres no son naturales. Lima, Perú: Red de Estudios Sociales en Prevención de Desastres en América Latina, 1993.

[4] E. Mansilla. Desastres modelo para armar: colección de piezas de un rompecabezas social. Lima, Perú: Red de Estudios Sociales en Prevención de Desastres en América Latina, 1996.

[5] A. Lavell. La gestión local del riesgo: nociones y precisiones en torno al concepto y la práctica. Guatemala, Guatemala: Centro de Coordinación para la Prevención de los Desastres Naturales en América Central [Cepredenac], Programa de las Naciones Unidas para el Desarrollo [PNUD], 2003.

[6] O. Cardona. Indicadores de riesgo de desastre y de gestión de riesgos: Pro- grama para América Latina y el Caribe; Informe Técnico Principal. Manizales, Colombia: Universidad Nacional de Colombia, 2005.

[7] O. Cardona, "La necesidad de repensar de manera holística los conceptos de vulnerabilidad y riesgo. Una crítica y una revisión necesaria para la gestión," en Int, Work-Conf. on Vulnerability in Disaster Theory and Practice, Wageningen, Holanda, 2001.

[8] 0. Cardona, "Estimación holística del riesgo sísmico utilizando sistemas dinámicos complejos," Tesis de doctorado, Escola Tècnica Superior D’Enginyers, de Camins, Canals i Port, Universitat Politècnica de Catalunya, Barcelona, 2001.

[9] Congreso de la República de Colombia, "Ley 1523 de 2012," en Diario Oficial 48411, p. 48, 2012.

[10] G. C. Gallopín, "Linkages between vulnerability, resilience, and adaptive capacity," Global Environmental Change, vol. 16, no. 3, pp. 293-303, 2006. https://doi.org/10.1016/j.gloenvcha.2006.02.004

[11] P. Blaikie, T. Cannon, L. David y B. Wisner. Vulnerabilidad: el entorno social, político y económico de los desastres. Lima, Perú: Red de Estudios Sociales en Prevención de Desastres en América Latina, 1996.

[12] C. Polsky, R. Neff y B. Yarnal, "Building comparable global change vulnerability assessments: The vulnerability scoping diagram," Global Environmental Change, vol. 17, no. 3, pp. 472-485, 
2007. https://doi.org/10.1016/j.gloenvcha.2007.01.005.

[13] D. Schroter, C. Polsky y A. G. Patt, "Assessing vulnerabilities to the effects of global change: an eight steop approach," Mitigation and Adaptation Strategies for Global Change, vol. 10, pp. 573-596, 2005. https://doi. org/10.1007/s11027-005-6135-9.

[14] Banco Mundial. Análisis de la gestión del riesgo de desastres en Colombia: un aporte para la construcción de políticas públicas. Bogotá, Colombia: Banco Mundial, 2012.

[15] Ministerio de Medio Ambiente, "Decreto 1729 de 2002", en Diario oficial 44893, p. 7, 2012.

[16] Instituto de Hidrología, Meteorología y Estudios Ambientales [Ideam]. Guía técnico científica para la ordenación de las cuencas hidrográficas en Colombia. Bogotá, Colombia: Instituto de Hidrología, Meteorología y Estudios Ambientales, 2008.

[17] Instituto de Hidrología, Meteorología y Estudios Ambientales [Ideam]. Guía técnico-científica para la ordenación y manejo de cuencas hidrográficas en Colombia. Bogotá, Colombia: Instituto de Hidrología, Meteorología y Estudios Ambientales, 2004.

[18] Colombia, Ministerio de Ambiente y Desarrollo Sostenible, "Decreto 1640 de 2012 ", en Diario oficial 48510, p. 28, 2012.

[19] Colombia, Ministerio de Ambiente y Desarrollo Sostenible. Guía técnica para la formulación de los planes de ordenación y manejo de Cuencas Hidrográficas POMCAS. Anexo B. Gestión del riesgo. Bogotá, Colombia: Ministerio de Ambiente y Desarrollo Sostenible, 2014.

[20] J. Vera y A. Albarracín, "Propuesta metodológica para el análisis y espacialización de la vulnerabilidad ante amenazas hidroclimáticas, para la gestión integral del riesgo en la planificación ambiental de cuencas hidrográficas," Tesis de maestría, Facultad de Ingeniería Forestal, Universidad del Tolima, Ibagué, Colombia, 2015.

[21] Colombia, Alcaldía de Ibagué. Estudio de caracterización para el ordenamiento del Corregimiento $N^{\circ}$ Nueve (9) del municipio de Ibagué. Ibagué, Colombia: Alcaldía de Ibagué, Secretaría de Planeación municipal, 2006.

[22] Cortolima. Plan de Ordenamiento de la Cuenca Mayor del Río Coello. Fases de diagnóstico y formulación. Ibagué, Colombia: Cortolima, 2006.

[23] Geotec Group. Estudio de amenazas naturales, vulnerabilidad y escenarios de riesgo en los centros poblados de Villa Restrepo, Llanitos, Juntas, Pastales, Pico de Oro, Bocatoma Combeima y Cay por flujos torrenciales en las Microcuencas del Rio Combeima. Ibagué, Colombia: Cortolima, 2013.

[24] C. Flórez, F. Espinoza y L. Sánchez. Diseño del índice SISBÉN en su tercera versión -SISBÉN III-. Bogotá, Colombia: Departamento Nacional de Planeación, 2008. 
[25] Colombia, Ministerio de Agricultura, "Decreto 1449 de 1977," en Diario Oficial No. 34827, p. 5, 1977.

[26] Congreso de la República de Colombia, "Decreto 2811 de 1974," en Diario Oficial No. 34243, 1974.

[27] Colombia Ministerio del Medio Ambiente, "Decreto 1791 de 1996," en Régimen de Aprovechamiento Forestal, 1996.

[28] L. Ocampo y L. Ramírez, “Determinación de la producción de sedimentos, bajo escenarios de cambio climático en la cuenca hidrográfica del río Coello, departamento del Tolima," Tesis de maestría, Facultad de Ingeniería Forestal, Universidad del Tolima, Ibagué, 2013.
[29] Transparencia por Colombia, "Índice de transparencia de las entidades públicas," 2016. [En línea]. Disponible en: http://indicedetransparencia.org.co

[30] Departamento Nacional de Planeación, "Evaluación del despeño fiscal de los entes territoriales," 2016. [En línea]. Disponible en: https://www.dnp.gov. co/programas/desarrollo-territorial/ evaluacion-y-seguimiento-de-la-descentralizacion/Paginas/desempeno-fiscal.aspx

[31] NASA, "ASTER Global Digital Elevation Map," 2011. [En línea]. Disponible en: https://asterweb.jpl.nasa.gov/gdem.asp 